Portland State University

PDXScholar

$1-1-1988$

\title{
Medium resolution atmospheric pressure ionization mass spectrometer
}

Andrew H. Grange

Robert J. O'Brien

Portland State University

Douglas F. Barofsky

Follow this and additional works at: https://pdxscholar.library.pdx.edu/chem_fac

Part of the Analytical Chemistry Commons

Let us know how access to this document benefits you.

\section{Citation Details}

Grange, A. H., O’Brien, R. J., \& Barofsky, D. F. (1988). Medium resolution atmospheric pressure ionization mass spectrometer. Review of Scientific Instruments, 59(4), 573.

This Article is brought to you for free and open access. It has been accepted for inclusion in Chemistry Faculty Publications and Presentations by an authorized administrator of PDXScholar. Please contact us if we can make this document more accessible: pdxscholar@pdx.edu. 


\title{
Medium resolution atmospheric pressure ionization mass spectrometer
}

\author{
Andrew $\mathrm{H}$. Grange \\ Department of Environmental Science and Engineering, Oregon Graduate Center, $19600 \mathrm{~N}$. W. Von \\ Neumann Drive, Beaverton, Oregon 97006 \\ Robert J. O'Brien \\ Department of Chemistry and Environmental Science Doctoral Program, Portland State University, Porland, \\ Oregon 97207 \\ Douglas F. Baroisky \\ Department of Agriculural Chemistry, Oregon State University, Corvallis, Oregon 97331 \\ (Received 29 June 1987; accepted for publication 5 January 1988)

\begin{abstract}
An atmospheric pressure ionization (API) source for a VG 7070E-HF mass spectrometer has been built and successfully operated at an accelerating potential of $6 \mathrm{kV}$. Hydrated protons and hydrated organic ions formed in the API source can be either partially or completely disaggregated by collisionally induced dissociation before entering the mass analyzer. The protonated molecular ions of pinacolone and 2,4-pentanedione can be fully separated ( $R=2800$, $m / z 101,<1 \%$ valley) at one sixth of their maximum signal strengths, and the ${ }^{13} \mathrm{C}$-xylene radical ion and protonated benzaldehyde can be fully separated ( $R=3300, \mathrm{~m} / z 107,<1 \%$ valley) at one tenth of their maximum signal strengths. With $1 \%$ transmission, the resolving power of the VG 7070E-HF operating in the API mode is estimated to be $4800(\mathrm{~m} / \mathrm{z} 92,10 \%$ valley $)$. This resolution is more than sufficient to separate two oxidized hydrocarbons whose masses differ by 0.0364 daltons, the difference between a $-\mathrm{CH}_{2} \mathrm{CH}_{2}-$ group and a $>\mathrm{CO}$ group, and to permit unambiguous assignment of their molecular formulas. No other form of API mass spectrometer, including the triple quadrupole version, has this capability.
\end{abstract}

\section{INTRODUCTION}

Atmospheric pressure ionization (API) mass spectrometry is sufficiently sensitive to identify and quantify analytes in the gas phase at trace concentrations undetectable by electron impact or chemical ionization methods. Detection limits at ppb to ppt levels are typical, and dynamic ranges of three or more decades are observable for many organic compounds. ${ }^{1}$ Hence, mixtures of trace components can, in principle, be studied in the gas phase over a wide range of concentrations without the time-consuming and potentially sample-altering prerequisites of concentration or separation that are necessary with less-sensitive ionization methods.

Atmospheric pressure ionization sources have most often been coupled with quadrupole mass fiters ${ }^{1-5}$ and, less often, with single-focusing magnetic sector mass analyzers. ${ }^{6,7}$ Whereas these instruments provide high sensitivity, they produce very low resolution mass spectra. Unit resolution is commonly cited for quadrupole systems; examination of published spectra suggests that in many cases this claim is optimistic. Sakairi and Kambara specified a resolution of 300 ( $10 \%$ valley) for their magnetic sector instrument operated with an acceleration voltage of $3 \mathrm{kV}$ (Ref. 8). Because of their low resolving powers, these instruments are incapable of separating the molecular ion of a species of interest from extraneous ions having the same nominal mass. Interfering ions can arise from other organic compounds present in the sample gas and from water clustering and fragmentation processes that take place in the ion source'; the problem is especially marked when numerous analytes are present in the sample gas.

We wish to study the oxidized products of certain hydrocarbons that are released into the atmosphere. This requires an instrument that at atmospheric concentrations separates two oxidized hydrocarbons whose masses differ by 0.0364 daltons, the difference between a $-\mathrm{CH}_{2} \mathrm{CH}_{2}-$ group and a $>\mathrm{CO}$ group, and permits their molecular formulas to be unambiguously assigned. This task requires an instrument with a resolving power of $\sim 4000$ (10\% valley) up to a molecular weight of 150 daltons. Since no existing form of API mass spectrometer, including the triple quadrupole version, had this capability, we undertook to build a source for a VG 7070E-HF mass spectrometer that would permit the instrument to be run in an API mode at a resolution approaching 5000. In this article we describe our API source's design, construction, and operation and demonstrate the mass spectrometer's resolution capabilities when operated with our source.

\section{SOURCE DESIGN AND CONSTRUCTION}

Ionization at atmospheric pressure for the purposes of mass spectrometry can be accomplished in various ways ${ }^{5}$; it is most often done by secondary ionization with beta particles emitted by ${ }^{63} \mathrm{Ni}$ foil or by corona discharge. ${ }^{2-5} \mathrm{We}$ chose to use the latter method because it offers a wider dynamic range, a modestly higher sensitivity, and the possibility of 
varying ion residence times within the region where they are created. $^{10}$

\section{A. Source block}

Construction details of our API source are displayed in Fig. 1 (note that Fig. 1 is not a uniplanar cross-sectional view of the source). The body of the source is machined from a 2 in.-diam, 304 stainless-steel cylinder. Shown in the figure are the sample inlet line, the corona discharge assembly, the first and second aperture assemblies, the pumping line, and the ion source's lens assembly; not shown are the vacuum gauge line provided to monitor the pressure in the collisionally induced dissociation (CID) region, the source heater (VG), and the thermocouple used to monitor the source temperature. The design of the source is based primarily on Caldecourt's, ${ }^{11}$ which, in fact, is a modification of a design by Kambara. 'Unlike Caldecourt's source, however, ours does not have a lens in the CID region; a more recent design by Kambara ${ }^{8}$ also lacks this lens.

The corona discharge assembly consists of a $5-\mathrm{kV}$ feedthrough (Ceramaseal), a shortened sewing needle made of Ni-coated steel (Milward 7 ), an A1-holder for the coronatip, and a Vespel cylinder that aligns the tip and its holder with the exit orifice. The Vespel cylinder has a $\frac{1}{4}$-in. milled slot that aing with the inlet port and allows unconstricted
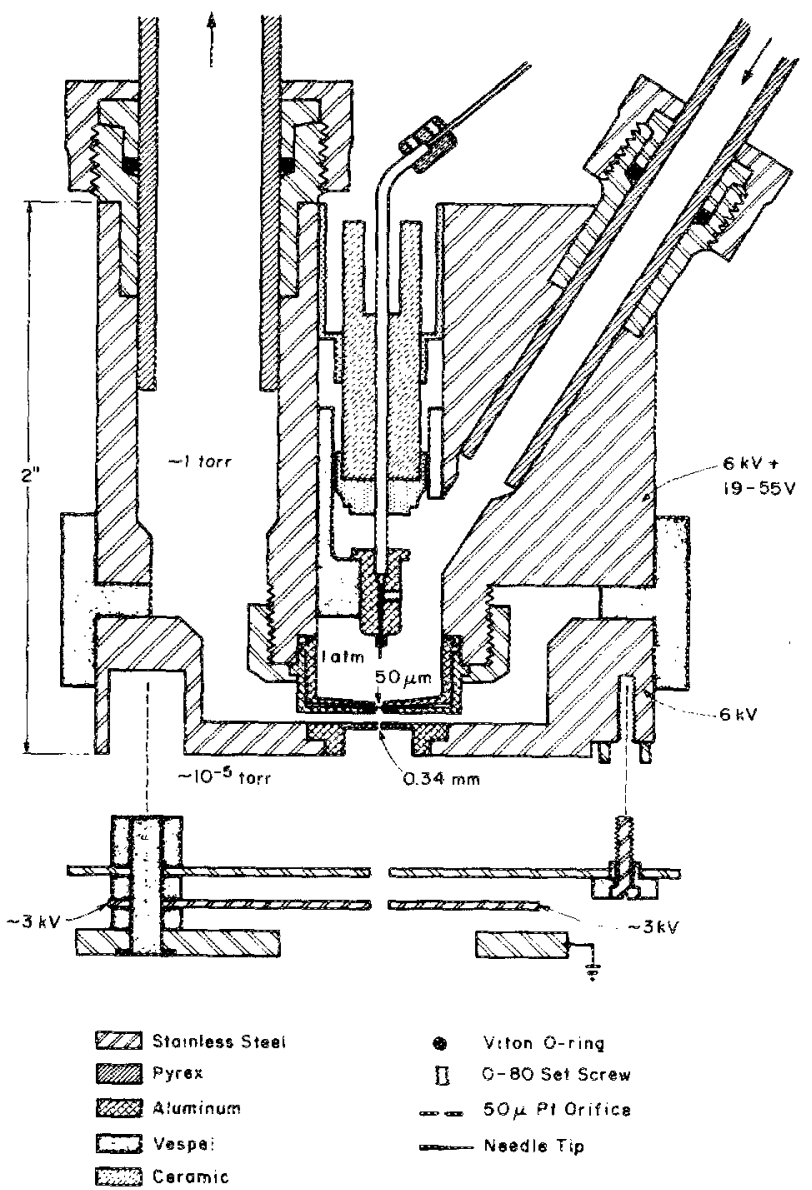

FIG. 1. Construction details of the API source (nonuniplanar cross section). flow of the sample gas into the corona discharge region. The tip of a corona needle is located $4.6 \pm 0.1 \mathrm{~mm}$ from the exit aperture by using a fixture to position the needle within its holder before tightening the set screw. This tip-to-orifice distance is $15 \%$ larger than that used by Dzidic et al. ${ }^{10}$; it was chosen to ensure sufficient residence time for the full sequence of ion-molecule reactions to take place and produce abundant, protonated analyte ions.

The circular aperture separating the corona region from the CID region is centered in a 3-mm-diam, 0.010-in.-thick Pt disk (Ebtec). The annular region surrounding the orifice is countersunk on one side of the disk so that the aperture itself is $-0.003 \mathrm{in}$. long. Operation of the source with four aperture sizes, viz., $25,50,75$, and $100 \mu \mathrm{m}$, was investigated. The aperture disk is located and held by two aluminum, cylindrical caps that are firmly clamped onto the end of the corona discharge chamber with a threaded ring (Fig. 1); the design allows quick access to the aperture disk for cleaning or replacement. The outside, bottom surface of the inner cap is faced at a $2^{\circ}$ angle to provide a pinching action on the aperture disk that ensures a good seal. The sealing surfaces of the present aluminum caps are too easily scratched; therefore, future replacement caps will be made of stainless steel. A Vespel ring provides the seal between the caps and the chamber body.

The 304 stainless-steel end plate of the API source is insulated from the source body by a large Vespel ring. This plate has an aluminum disk with a $0.34-\mathrm{mm}$ aperture pressfitted into its center and a standard VG ion source lens assembly mounted onto its outside face. The Macor standoffs normally used to separate the lens electrodes were replaced with more durable Vespel insulators. The last lens plate, which is maintained at ground potential, has two holes which mate to dowels located inside the source housing on the support structure just in front of the entrance slit assembly; this docking arrangement, which is a standard feature of commercial VG ion sources, automatically aligns the axis of the ion source with the optical axis of the mass analyzer.

\section{B. Source support}

A customized support assembly was built to adapt the API source to the VG 7070 E-HF mass spectrometer. The design preserves the standard, modular construction of commercial ion sources built for VG 7070 mass spectrometers. The main support components were custom fabricated from 304 stainless steel; the API source is insulated from these components by three Vespel posts. Three gas lines service the API source: an iniet line that provides the corona discharge region with sample gas at 1 atm, a line leading from the CID region to a Teledyne-Hastings-Raydist DV-6M TC pressure gauge outside the vacuum envelope (not shown in Fig. 1), and a large diameter, Pyrex pumping line leading from the CID region. Both Teflon and glass-metal bellows have been employed for the 0.25-in.-o.d. sample-inlet and vacuum gauge lines. Either form provides adequate insulation for the source, which is maintained at $6 \mathrm{kV}$, and at the same time ample flexibility to accommodate the slight movement of the outer flange relative to the source during pump down. A 1-in.-o.d. metal bellows seaied to the Pyrex pro- 


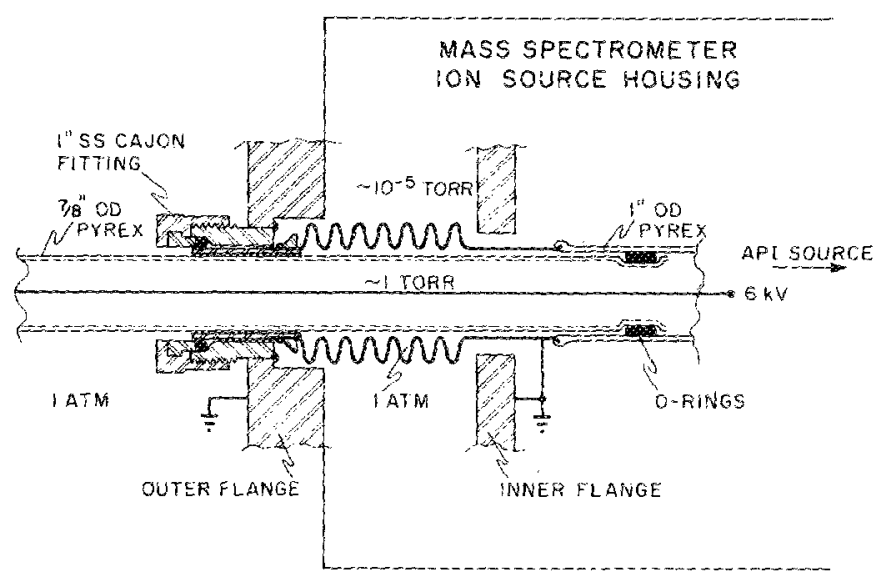

FrG. 2. Detail of the API source's pumping line.

vides this same function for the large pumping line (Fig. 2); the integrity of the vacuum envelope is maintained at the point where the purnping line passes through the sourcehousing flange by a 1 -in. Cajon O-ring fitting welded in to the flange. Similar seals with 0.25 -in. Cajon 0 -ring fitings are provided for the sample inlet line and the pressure measurement line at their points of egress.

Eleven $10-\mathrm{kV}$ feedthroughs (Ceramaseal) are welded into the source-housing flange to provide electrical connections to the APl source, beam centering half-plates, thermocouple junction, and heater; the feedthroughs were modified to conform to standard VG form and dimensions.

\section{Pumping system and discharge suppression}

A 50-cfm, Alcatel 2063, two-stage rotary pump is used to pump the APl source. During operation, the pressure within the pumping line falls from -1 Torr in the CID region to $\sim 0.05$ Torr at the head of the pump. In the vicinity of 0.1 Torr, conditions are ideal for glow discharges between the API source at $6 \mathrm{kV}$ and grounded surfaces along the pumping line ${ }^{12}$; these discharges disrupt operation, damage electronic components, and expose human operators to the hazard of a high-voltage shock. The arcing problem was eliminated by devising a discharge suppression system ${ }^{13}$ to inhibit arcing along the pumping line to the pump and by preventing grounded strfaces within the mass spectrometer's ion source housing from being exposed to high-voltage elements of the source through conducting gas volumes. Figure 2 shows how the large, grounded metal bellows used in the main pumping line was isolated from the gas within the pumping line ( -1 Torr $)$; the volume of gas extending from the three Buna-N O-rings between the inner surface of the bellows and the outer surface of the inner Pyrex tubing is at atmospheric pressure and is nonconducting at ion source potentials $(\leqslant 6 \mathrm{kV})$.

\section{Voltage supplies}

The corona discharge is initiated and maintained by a positive, $15-\mathrm{kV}$ power supply (Bertan model 602B-150P) modifed to provide a constant current; the discharge is monitored with an ammeter (Hickok LX 303 DMM) placed in series with the external high-voltage connection to the corona discharge tip. A limiting resistance of $500 \mathrm{M} \Omega$ is also in series with the high-voltage comection to the tip in order to protect the circuit from sporadic arcing.

The voltage applied to the end piate of the API source (6-kV accelerating potential) and the voltages applied to the half-plates of the source lens (focusing and beam centering potentials, $\sim 3 \mathrm{kV}$ ) are provided by an independent, positive, $10-\mathrm{kV}$ power supply (Bertan model 602B-100P), instead of the mass spectrometer's ion source supply, to protect against the unlikely chance of arcinduced damage to the electronics and data-acquisition system.

The potential across the CID region is provided by a voltage supply consisting of six $9-\mathrm{V}$ batteries in series and a potentiometer that can be switched across any battery in the string; the high-resistance side of the potentiometer is connected to the switch across a $1.5-\mathrm{V}$ battery. The output of this supply can be varied continuousiy from $0-56 \mathrm{~V}$.

\section{SOURCE OPERATION AND PERFOAMANCE}

\section{A. General}

Except where otherwise indicated, room air admitted into the source through the open inlet port was used for testing (the limiting conductance was provided by the aperture separating the corona discharge and CID regions). The source is started by switching on the corona discharge power supply to $8-9.5 \mathrm{kV}$ (measured from the discharge tip to ground). Interruption of the ion signal, especially when a new reedle tip is used, occurs intermittently for the first few minutes following initiation of the corona discharge; to reduce the time necessary to achieve stable cperation, the discharge is run at $1-1.5 \mu \mathrm{A}$ for about 2 min. Following this conditioning period, the discharge potential is adjusted to produce an average operating current of $0.2 \mu \mathrm{A}$. During corona discharge, the needle tips are blunted by sputtering and are replaced when the ion signals become erratic. This effect was found to be minimized by operating at the lowest sustainable corona discharge current for our source $(0.2 \mu \mathrm{A})$.

After operating the corona discharge continuously for $\sim 30$ min, the discharge current drifts by only $\pm 0.01 \mu \mathrm{A}$ during the time required to acquire a single mass spectrum $(\sim 2 \mathrm{~min})$ and by only $\pm 0.03 \mu \mathrm{A}$ during periods $\geqslant 1 \mathrm{~h}$. Peak heights fluctuate by as much as $\pm 20 \%$ (time constant $=0.1 \mathrm{~ms}$ ); these fluctuations are most pronounced when low corona discharge currents are used. To compensate for the short-term variations in ion beam intensities and obtain good average peak areas, which appear as lines after the peaks are certroided by the data-acquisition system, all mass spectra were recorded at the slow scan rate of 110 s per mass decade. The average lifetime for corona discharge tips operated at $0.2 \mu \mathrm{A}$ under relatively clean conditions is estimated to be $\sim 10 \mathrm{~h}$. If there are high levels of organic com pounds in the sample, deposits form on the corona tip and operation becomes erratic after only a few hours. Corona discharge tips made from inidium wire might have greater lifetimes ${ }^{11}$; however, given the ease with which tips can be changed in our source, the short conditioning time required for new tips, and the low cost of sewing needles, the relatively 
short lifetimes of discharge tips has not been cause for concern.

Comparison tests with 25-, 50-, 75-, and 100- $\mu \mathrm{m}$ apertures revealed that the 50 - and $75-\mu \mathrm{m}$ orifices produced the most intense total ion beams. It was decided to use the $50-\mu \mathrm{m}$ aperture routinely because of its lower conductance, a matter of concern when samples are supplied from finite sources, such as photoreaction chambers. The gas flow through the API source with a well-sealed $50-\mu \mathrm{m}$ aperture in place is $18-$ $21 \mathrm{~cm}^{3} / \mathrm{min}$. Clogging of the corona region's aperture by sputtered material has not been observed at the tip-to-orifice distance and low corona currents normally used. On a few occasions, however, particles carried by the sample gas stream have become lodged in the 50 - $\mu \mathrm{m}$ orifice. When this occurs, the source is removed and disassembled; the orifice is cleaned using ultrasonification; the source is reassembled and reinserted; and the mass spectrometer source region is reevacuated, all in less than $30 \mathrm{~min}$. The replacement of corona discharge needles also requires less than $30 \mathrm{~min}$.

When the API source heater is used, large peaks arising from phthalates, e.g., protonated phthalic anhydride at $m / z$ 149 , appear in the mass spectra. We atiribute the appearance of these peaks to the Vespel components in the source assembly. These parts will be made of Macor or Cu as necessary in a future version of the source.

The alignment provided by the discharge tip assembly, the aperture holders, and the source's locating doweis is so reproducible that only subtie, if any, adjustment of the potentials on the beam-centering plates or those that control the $y$ and $z$ directions of the beam path in the mass analyzer are required after removal, cleaning, reinsertion, and pump down of the API source (slits set for $\sim 90 \%$ of maximum peak height).

\section{B. Deciustering}

When air is used as the matrix for a sample gas, a rapid sequence of ion-molecule reactions occurs in the corona discharge leading to formation of hydrated protons, $\mathrm{H}\left(\mathrm{H}_{2} \mathrm{O}\right)_{n}{ }^{+}(n \geqslant 1) .^{14,15}$ These cluster ions serve as the primary reagent ions for positive ionization since trace molecules that are stronger gas-phase bases than water will be protonated by the hydronium ion or higher-order clusters. Many classes of organic compounds containing heteroatoms, e.g., $O$ or $N$, have sufficiently high proton affinities to be ionized via this gas-phase ion-molecule reaction. Hydrated ions formed from these compounds are also observed at the mass analyzer's detector. The origin of these hydrated species can be rationalized on at least two bases: (1) transfer of one or more of water molecules during protonation ${ }^{3}$ and (2) condensation of water molecules onto ions during adiabatic expansion of the gas stream through the orifice exiting the discharge region. ${ }^{16}$

It is desirable to find operating conditions that coalesce each series of hydrated ions into only one or two intense peaks and thereby simplify the mass spectra. Kambara and Kanomata demonstrated that declustering can be accomplished through collisionally induced dissociation by applying a potential across the differentially pumped region between the two orifices when this region is maintained at a pressure of $\sim 1$ Torr. ${ }^{17}$ The cluster ions are accelerated by the applied potential and acquire kinetic energy that is apparently transferred into their vibrational modes during collisions with neutral molecules. After a sufficient number of collisions, a cluster ion loses an attached water molecule; depending on the applied CID potential, the width of the CID region, and the number density of neutral molecules
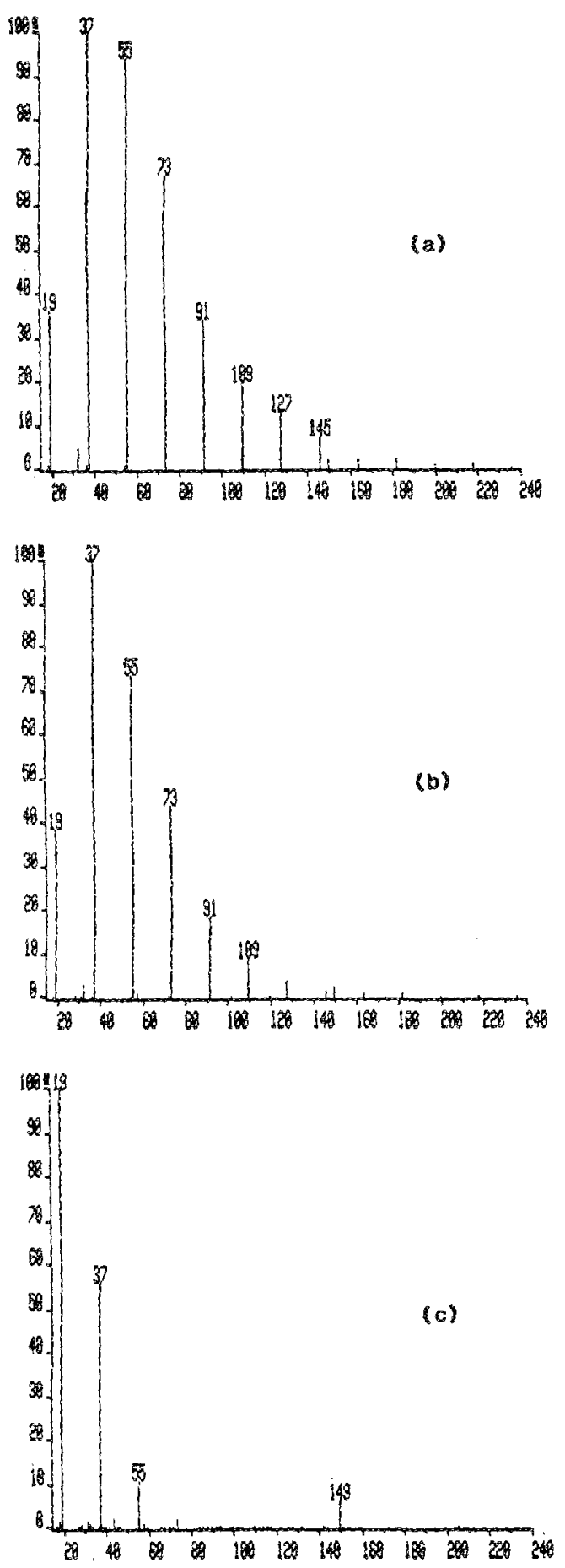

FIG. 3. API mass spectra of room air at $40+15 \%$ relative humidity recorded at CID pressure/potential combinations of (a) $0.4 \mathrm{Tor} / 19 \mathrm{~V}$, (b) 1.0 Torr $/ 19 \mathrm{~V}$, and (c) 1.0 Torr $/ 55 \mathrm{~V}$. The mass peak observed in spectrum (c) at $m / z 149$ corresponds to protonated phthalic anhydride. 
present, this process might be repeated until only a hydronium ion remains.

A set of mass spectra of room air at a relative humidity of $40 \pm 15 \%$ was recorded to observe the effects of the CID region's pressure and the CID potential on the abundarices of the hydrated protons. CID potentials were varied in $9 . \mathrm{V}$ increments from 19 to $55 \mathrm{~V}$ for each of three ClD pressures, viz., 1.0, 0.7, and 0.4 Torr. Statistical analyses of the fuctu: ations in the heights of the five largest peaks in the entire set of mass spectra were made to ensure that the phenomena observed were not due to either systematic or random insta. bilities in some region of the ion source. "The mass spectra in Fig. 3 illustrate the trends in ion formation and cluster disaggregation that occur under different extremes of CID pressure and potential. The range of pressures in the $\mathrm{CHD}$ region was constrained at the low end by sporadic arcing and at the high end by the limited pumping speed of the mass spectrometer's oil diffusion pumps. To eliminate the possibility of sporadic arcing, all but the experiment represented in Fig. 3(a) were performed using a CID pressure of 1 Torr. Figure $3(\mathrm{a})$ exhibits a mass spectrum recorded at the lowest settings of CID pressure and CID potential studied; the distribution of hydrated protons $(m / z=19,37,55,73, \ldots, 235$ corresponding to $\left.\mathrm{H}\left(\mathrm{H}_{2} \mathrm{O}\right)_{n}{ }^{+}, 1 \leqslant n \leqslant 13\right)$ cleariy shows that the higherorder clusters are abundant under these conditions. Increasing the CID pressure by over a factor of 2 causes only a minor decrease in the abundances of the higher-order clusters [Fig. 3(b)], whereas increasing the CID potential to 55 $\mathrm{V}$, nearly a factor of 3 , clearly produces marked declusiering of the hydrated protons [Fig. 3(c)]. Consequently, we use only the CID potential to control declustering in the CID region.

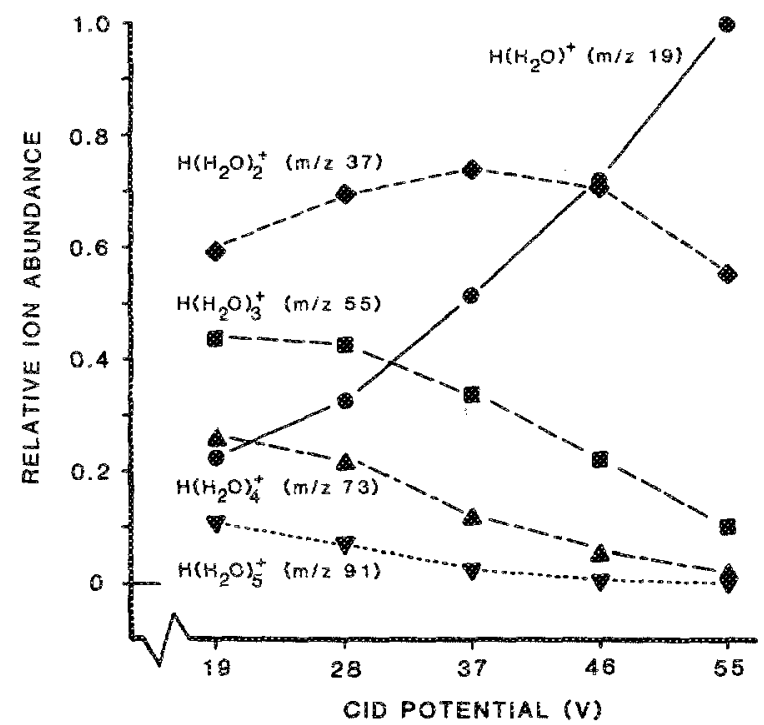

FiG. 4. Mlots of relative ion abundance at a CID pressure of 1.0 Torr vs CID potential. Relative fon abundance is defined by the

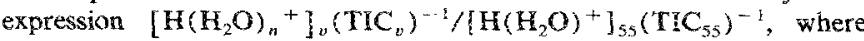
$\left[\mathrm{H}\left(\mathrm{H}_{2} \mathrm{O}\right)_{n}{ }^{+}\right]_{e}$ and $\mathrm{TIC}_{v}$ are, respectively, the peak area of $\mathrm{H}\left(\mathrm{H}_{2} \mathrm{O}\right)_{n}{ }^{+}$ recorded at the CID potential $V$ and the total ion current for the mass spectrum in which this peak appears. Each peak area is normalized with respect to the total ion current of its corresponding mass spectrum in order to compensate for any differences in ion collection efficiency between recordings.
Figure 4 shows the relative ion abundances of the first five hydrated protons plotted as a function of CID potential; the relative ion abundances were computed from tabular listings of the entire set of room air mass spectra. As the CID potential increases, declustering becomes more complete, and the larger hydrated protons $(m / z 55,73$, and 91$)$ fall in abundance relative to that of the hydronium ion $(\mathrm{m} / z 19)$ at $55 \mathrm{~V}$. The abundance of the hydronium ion itself increases steadily up to its maximum at the CID potential of $55 \mathrm{~V}$. The relative abundance of $\mathrm{H}\left(\mathrm{H}_{2} \mathrm{O}\right)_{2}+(\mathrm{m} / \mathrm{z} 37)$ increases and then falls with increasing CID potential. This behavior reflects the competition between disaggregation of $\mathrm{H}\left(\mathrm{H}_{2} \mathrm{O}\right)_{2}$, while passing through the CID region and formation of $\mathrm{H}\left(\mathrm{H}_{2} \mathrm{O}\right)_{2}{ }^{+}$via declustering of larger hydrated protons. At the highest CID potential, spectra are simplest as can be seen by comparing Figs. 3(a) with 3(c).

A high concentration of an easily protonated compound in the sampled air can decrease the degree of hydronium ion clustering. This effect is illustrated in Fig. 5 for the case of benzaldehyde vapor; the average cluster size, $\vec{n}$, is 2.92 in
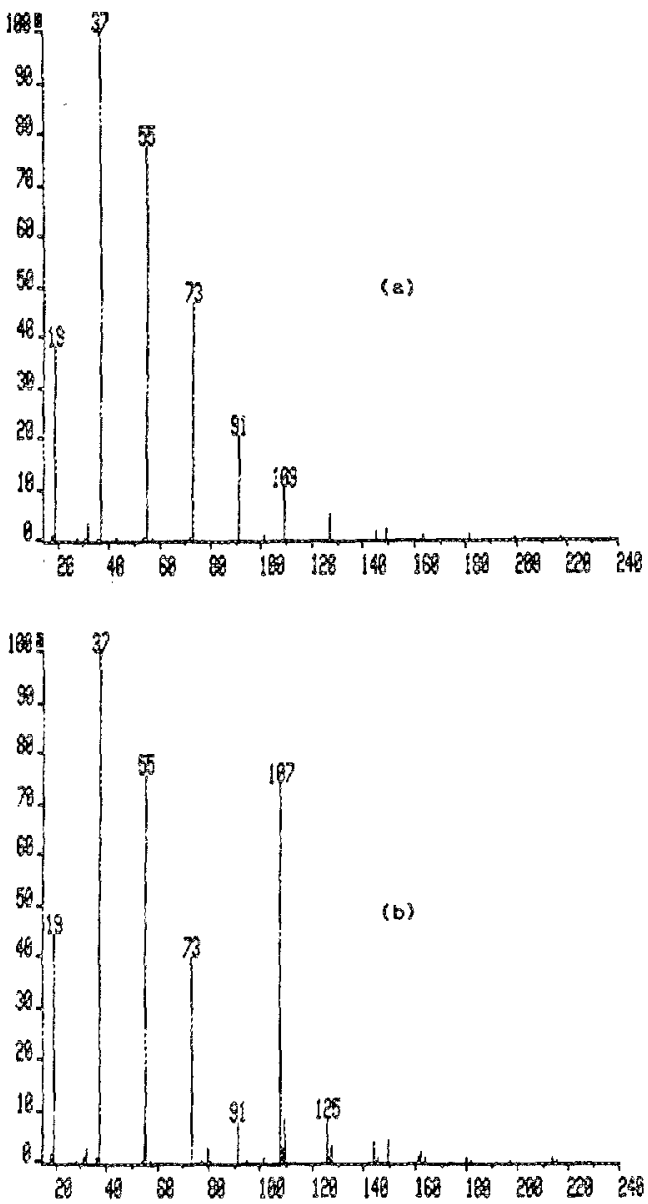

FIG. 5. A.PI mass spectra of (a) room air and (b) room air with benzaldehyde vapor entrained into the inlet stream by suspending a small beaker containing several drops of the liquid near the sample inlet port. Both speetra were recorded with the CID potential at $19 \mathrm{~V}$ and the CID pressure at 1.0 Torr to ensure the presence of numerous water chuster ions. The $m / z 10$ ? peak corresponds to protonated benzaldehyde; the $m / 2125,143$, and 161 peaks correspond to hydrated, protonated benzaldehyde ions; and the $m / z$ 213 peak corresponds to protonated bencaldehyde dimer. 
Fig. 5(a) and $2.71 \mathrm{in}$ Fig. 5(b). The degree of declustering is obviously small relative to that induced by increasing the CID potential, and it virtually disappears at low levels of analyte. In the same manner as for the hydrated protons, the abundances of the hydrated, protonated benzaldehyde ions and of the protonated benzaldehyde dimer ion decrease as the CID potential increases.

The effects of diluting samples with dry zero air and of varying pressure in the corona region on the formation of cluster ions, as well as the effect of the CID potential on disaggregating cluster ions, are more completely reported and discussed in a companion paper. ${ }^{9}$

\section{Resolution}

To determine the resolving power of the API-VG $7070 \mathrm{E}$ mass spectrometer operating in the API mode, several mass spectra of various doublets in the vicinity of 100 daltons were recorded; the scans were made from high to low mass over a restricted range of approximately 5 daltons. The CID voltage was adjusted to maximize the peak height of the nominal mass being resolved. The base resolution of the instrument, defined as that with the entrance and exit slits of the mass analyzer set to give an ion signal at a given mass-tocharge ratic equal to $\sim 90 \%$ of its maximum, was determined from ten consecutive scans over the protonated benzaldehyde ions, ${ }^{12} \mathrm{C}_{7} \mathrm{H}_{7} \mathrm{O}^{t-}$ and ${ }^{12} \mathrm{C}_{6}{ }^{13} \mathrm{CH}_{7} \mathrm{O}^{+1}$, to be 1525 $\pm 90(2 \sigma), 10 \%$ valley. A preliminary plot of standard additions of benzaldehyde indicates a minimum detectable limit for one scan ( $200 \mathrm{~s} /$ decade) at base resolution of $8 \mathrm{ppb}$ in air. ${ }^{18}$

A test atmosphere consisting of $1 \mathrm{ppm}$ of pinacolone (3,3-dimethyl-2-butanone), 3 ppm of 2,4-pentanedione, and zero air at a relative humidity of $50 \%$ was prepared in a $12-1$ flask, diluted $1: 8$ with dry zero air, and introduced into the API source. The CID potential was tuned to $27 \mathrm{~V}$ to maximize the molecular ion, i.e. $(\mathbf{M}+\mathbf{H})^{+}$, signals. In order to completely separate the two mass peaks at $m / z 101$, the entrance slit was first narrowed to reduce the ion signal from its maximum by a factor of 3 , and then the exit sit was narrowed to further reduce the ion signal by an additional factor of 2 . Figure 6 is a typical mass spectrum of the fully resolved protonated ions of pinacolone and 2,4-pentanedione. The very large signal-to-noise ratio clearly indicates that these two species could be separated at much lower concentrations. The average valley for ten successive scans was less than $0.5 \%$, and the average ratio of peak heights for the same ten scans was 1:0.96. Hence, the resolution at one sixth of the open-slit signal strength is $2800(101 / 0.03639)$.

The mass spectrum in Fig. 7 shows the fully resolved doublet at $\mathrm{m} / \mathrm{z} 107$ comprised of xylene radical ion containing one ${ }^{13} \mathrm{C}$ atom and protonated benzaldehyde. A test atmosphere containing $1 \mathrm{ppm}$ of benzaldehyde was supplied to the API source in the same manner described for the previous doublet. A CID potential of $28 \mathrm{~V}$ maximized the benzaldehyde ion signal, and the entrance slit was narrowed to reduce the maximum ion signal to one fifth before narrowing the exit slit to reduce the remaining signal by one half. Sensitivity for xylene, which is devoid of heteroatoms, is extreme-

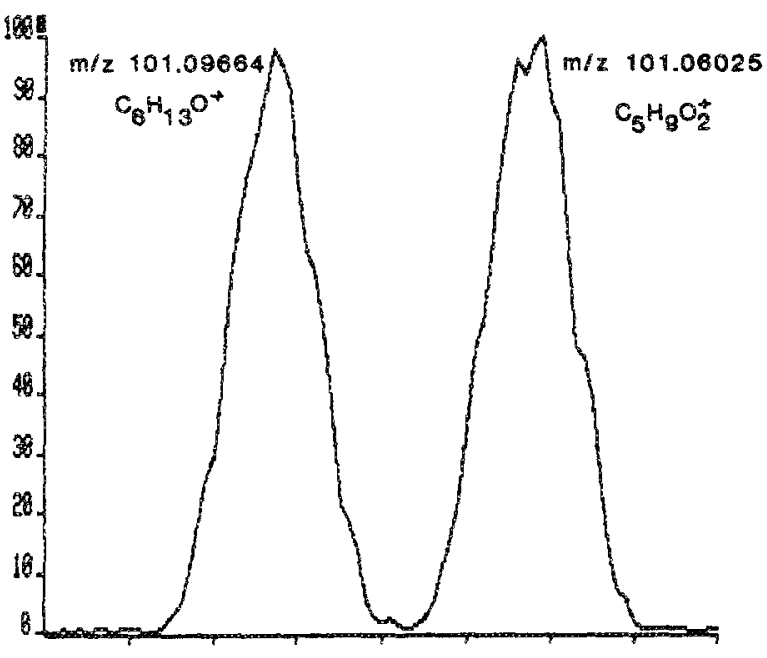

Fic. 6. API mass spectrum showing protonated pinacolone $\left(\mathrm{C}_{6} \mathrm{H}_{13} \mathrm{O}^{+}\right.$, $\mathrm{m} / \mathrm{z}$ 101.09664) fully resolved from protonated 2,4-pentanedione $\left(\mathrm{C}_{5} \mathrm{H}_{9} \mathrm{O}_{2}{ }^{+}, m / z 101.06025\right) ; R=2800,<0.5 \%$ valley.

ly low. To provide the ${ }^{13} \mathrm{C}$-xylene signal, a $0.02-\mu 1$ droplet was injected into the sample inlet line. As the sample atmosphere slowly became saturated with xylene, benzaldehyde's molecular ion abundance fell; both peaks became equal in height at about $15 \%$ of the original benzaldehyde ion signal. The lower ion abundances account for the greater noise observed in Fig. 7 relative to that seen in Fig. 6 . The average valley, which was determined from ten specira (two each from five separate injections of xylene), was found to be less than $0.5 \%$; thus, the resolution at one tenth of the open-slit signal strength is $3300(107 / 0.03192)$.

Toluene was admitted into the system in an attempt to separate the tropylium ion, $\mathrm{C}_{7} \mathrm{H}_{7}+(\mathrm{m} / \mathrm{z}$ 91.05478) from the hydrated proton $\mathrm{H}\left(\mathrm{H}_{2} \mathrm{O}\right)_{5}+(\mathrm{m} / z 91.06065)$, a task requiring a resolution $\sim 1500$. For this test the entrance slit was set to allow transmission of one tenth of the maximum signal; the remaining signal was successively halved by each of the two $z$ restrictors located in the flight tube and the exit slit, leaving the signal at $\sim 1 \%$ of its maximum. The width of

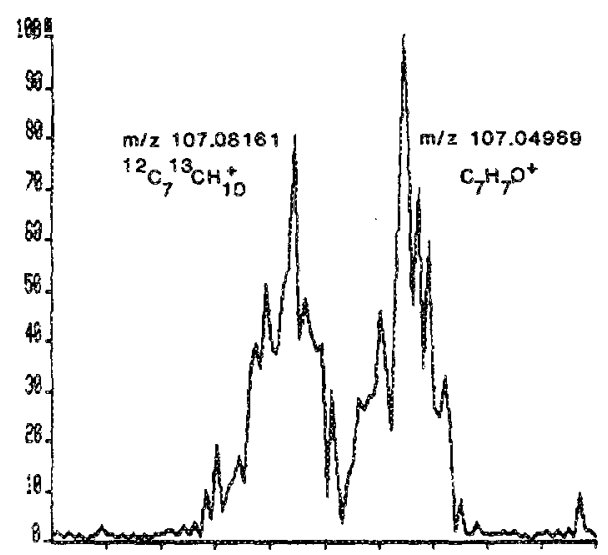

FIG. 7. API mass spectrum showing ${ }^{13} \mathrm{C}$-xylene radical ion $\left({ }^{12} \mathrm{C}_{7}{ }^{13} \mathrm{CH}_{10}{ }^{4}\right.$, $m / z 107.08161)$ fuily resolved from protonated benzaldehyde $\left(\mathrm{C}_{7} \mathrm{H}_{7} \mathrm{O}^{+}\right.$, $m / z$ 107.04969); $R=3300,<0.5 \%$ valley. 
toluene's molecular ion peak at $m / z 92$ was determined from the time-based units provided by the data-acquisition system to be 0.019 daltons $5 \%$ above the base line corresponding to a resolution of $\sim 4800(92 / 0.019)$ at $10 \%$ valley. This performance meets our design criterion, and we have already successfully used the instrument to assign molecular formulas to two, previously unresolvable photo-oxidation products of tolwene generated in an irradiated smog chamber. ${ }^{19}$

\section{ACKNOWLEDGMENT}

This work was supported by the Environmental Protection Agency, Grant No. R811876.

${ }^{1}$ N. M. Reid, J. B. French, J. A. Buckley, D. A. Lane, A. M. Lovett, and G. Rosenblatt, in Proceedings of the 4th Joint Conference on Sensing Environmental Pollutants (American Chemical Society, Washington, DC, 1978), pp. 594-600.

${ }^{2}$ R. K. Mitchum and W. A. Korfmacher, Anal. Chem. 55, 1485A (1983).
${ }^{3}$ C. J. Proctor and J. F. J. Todd, Org. Mass Spectrom. 18, 509 (1983). 'E. C. Horning, D. I. Carroll, I. Dzidic, K. D. Haegele, M. G. Horning, and

R. N. Stillwell, in Advances in Mass Spectrometry in Biochemistry and Medicine, edited by A. Frigerio and N. Castagnoli (Spectrum, Holliswood, New York, 1976), Vol. I, p. 1.

${ }^{5}$ M. McKeown and M.W. Siegel, Am. Lab. 89 (Nov. 1975).

'Y. Yinon and H. G. Boettger, Vacuum 26, 159 (1976).

${ }^{7} \mathrm{H}$. Kambara and I. Kanomata, Anal. Chem. 49, 270 (1977).

${ }^{8}$ M. Sakairi and H. Kambara, Mass Spectrom. 31, 87 (1983).

${ }^{9}$ A. H. Grange, R. J. O'Brien, and D. F. Barofsky (to be published).

${ }^{10}$ l. Dzidic, D. I. Carroll, R. N. Stillwell, and E. C. Horning, Anal. Chem. $\$ 8,1763(1976)$

${ }^{1}$ V. J. Caidecourt, D. Zakett, and J. C. Tou, Int. J. Mass Spectrom. Ion Phys. 49, 233 (1983).

${ }^{12}$ J. H. Futrell and L. H. Wojcik, Rev. Sci. Instrum, 42, 244 (1971).

1.A. H. Grange, R. J. O'Brien, and D. F. Barofsky, Rev. Sci. Instrum. 59, $656(1988)$.

${ }^{14}$ A. Good, D. A. Durden, and P. Kebarle, J. Chem. Phys. \$2, 212 (1970).

${ }^{15}$ A. Good, D. A. Durden, and P. Kebarle, J. Chem. Phys. 52, 222 (1970).

16P. Kebarle, \$. K. Searies, A. Zolla, J. Scarborough, and M. Arshadi, J. Am. Chem. Soc. 89, 6393 (1967).

${ }^{17}$ H. Kambara and I. Kanomata, Int. J. Mass Spectrom. Ion Phys. 25, 129 (1977).

${ }^{18}$ A. H. Grange, Ph.D. thesis, Oregon Graduate Center, 1988.

${ }^{19}$ A. H. Grange, R. J. O'Brien, and D. F. Barofsky (to be published). 